\title{
Cuidado de enfermería a la persona con estenosis aórtica \\ severa posterior al implante valvular aórtico transcatéter ${ }^{1}$
}

Mariana Martínez Borja²

Sandra Sonalí Olvera-Arreola ${ }^{3}$

doi:10.11144/Javeriana.IE17-1.cepe

Cómo citar: Martínez Borja M, Olvera-Arreola SS. Cuidado de enfermería a la persona con estenosis aórtica severa posterior al implante valvular aórtico transcatéter. Investig Enferm. Imagen Desarr. 2015;17(1):45-64. http://dx.doi.org/10.11144/Javeriana.IE17-1. cepe

1. Artículo de revisión. Recibido: 4 de abril de 2014. Revisado: 5 y 6 de mayo de 2014. Aceptado: 21 de julio de 2014.

2. Pasante de la Licenciatura en Enfermería y Obstetricia, Escuela Nacional de Enfermería y Obstetricia, Universidad Nacional Autónoma de México. Correo electrónico: mariana. borja37@gmail.com

3. Maestra en Enfermería. Jefa del Departamento de Investigación en Enfermería, Instituto Nacional de Cardiología Ignacio Chávez. Correo electrónico: sandra.olvera@cardiologia. org.mx 


\section{Resumen}

Introducción: Puesto que el pronóstico de la estenosis aórtica es desalentador, el 90\% de estas personas presenta síncope, angina o insuficiencia cardiaca, que ocasiona la muerte en un periodo de dos a tres años, en promedio. Los implantes valvulares aórticos transcatéter (TAVI) surgen como alternativa de tratamiento para este grupo de pacientes con riesgo quirúrgico elevado, en la octava década de vida, que presenten otras comorbilidades como enfermedades hepáticas, renales u otras, además de aquellos que no son candidatos para realizarles la cirugía cardiaca convencional. El presente artículo describe los antecedentes históricos del TAVI, sus indicaciones y contraindicaciones, las complicaciones del implante, así como el desarrollo del procedimiento, haciendo hincapié en una serie de intervenciones propuestas para que el profesional de enfermería otorgue cuidados seguros y de calidad, basados en evidencia científica. Objetivo: Describir las características generales del TAVI, así como proponer cuidados de enfermería al paciente sometido a este procedimiento durante el periodo inmediato y mediato. Metodología: Búsqueda sistematizada en bases de datos PubMed, EMBASE, SciELO, Medigraphic, Biblioteca Virtual en Salud. Se buscaron artículos originales, revisiones sistematizadas y metanálisis escritos en inglés, español y portugués, del 2007 al 2013. Conclusión: El TAVI ofrece una opción más de tratamiento a este grupo de pacientes; es importante que el profesional de enfermería posea conocimientos sobre los cuidados específicos que hay que ofrecer para que la recuperación sea rápida y las intervenciones sean oportunas y seguras.

Palabras clave: válvula-aórtica; estenosis-aórtica; cuidados de enfermería; valvuloplastía con balón; cateterismo cardiaco

\section{Nursing Care for the Person with Severe Aortic Stenosis after the Transcatheter Aortic Valve Implantation}

\section{Abstract}

Introduction: The prognosis for aortic stenosis is grim, $90 \%$ of the patients presented syncope, angina or heart failure, which causes death within two to three years, on average. The transcatheter aortic valve implant (TAVI) emerge as an alternative treatment for this group of patients at high surgical risk, in the eighth decade of life, who have other comorbidities such as liver disease, kidney or other, plus those who are not candidates for conventional cardiac surgery. This article describes the historical background of TAVI, its indications and contraindications, complications of implant and procedure development, focusing on a number of proposed interventions for the nurse to give safe, quality care based on scientific evidence. Objective: To describe the general characteristics of TAVI, and propose nursing care of the patient undergoing this procedure for the immediate and medium term. Methods: A systematic search in PubMed, EMBASE, SciELO, Medigraphic, and Biblioteca Virtual en Salud. The search included original articles, systematized reviews and meta-analysis in English, Spanish and Portuguese from 2007 to 2013.Conclusion: TAVI offers another option for treating this group of patients; it is important that the nurse have knowledge of specific care to be offered for the recovery to be quick and interventions to be timely and secure.

Keywords: aortic-valve; aortic stenosis; nursing care; balloon valvuloplasty; cardiac catheterization 


\section{Cuidado de enfermagem à pessoa com estenose aórtica severa posterior ao implante valvar aórtico transcateter}

\section{Resumo}

Introdução: Dado que o prognóstico da estenose aórtica é desalentador, $90 \%$ dessas pessoas apresenta síncope, angina ou insuficiência cardíaca, que ocasiona morte em um período de dois para três anos, como média. O implante valvar aórtico transcateter (TAVI) surge como alternativa de tratamento para esse grupo de pacientes com risco cirúrgico elevado, na oitava década de vida, que apresentem outras comorbidades como doença hepática, renal ou outras, além de aqueles que não são candidatos para lhes realizar a cirurgia cardiaca convencional. O presente artigo descreve os antecedentes históricos do TAVI, suas indicações e contraindicações, as complicações do implante, assim como o desenvolvimento do procedimento, enfatizando em uma série de intervenções propostas para que o profissional de enfermagem outorgue cuidados seguros e de qualidade, baseados em evidencia científica. Objetivo: Descrever as caraterísticas gerais do TAVI, assim como propor cuidados de enfermagem ao paciente sometido a esse procedimento durante o período imediato e mediato. Metodologia: Procura sistematizada em bases de dados PubMed, EMBASE, SciELO, Medigraphic, Biblioteca Virtual em Saúde. Procuraram-se artigos originais, revisões sistematizadas e meta-análise escritos em inglês, espanhol e português, do 2007 para 2013. Conclusão: O TAVI oferece uma opção mais de tratamento a esse grupo de pacientes; é importante que o profissional de enfermagem possua conhecimentos sobre os cuidados específicos que tem de oferecer para que a recuperação seja rápida e as intervenções oportunas e seguras.

Palavras-chave: valva-aórtica; estenose-aórtica; cuidados de enfermagem; valvoplastia por balão; cateterismo cardíaco 


\section{Introducción}

Con el paso del tiempo, la tecnología va avanzando y los procedimientos van mejorando. Así, el implante valvular percutáneo transcatéter (TAVI, por su sigla en inglés) es la alternativa de tratamiento menos invasiva para la estenosis valvular aórtica (EA) en aquellos pacientes de edad avanzada con otras comorbilidades agregadas. Pese a que la bibliografía médica en relación con esta temática es amplia, se observa un vacío en la producción de enfermería; por lo tanto, este manuscrito tiene como objetivo describir las características generales del TAVI, así como proponer cuidados al paciente sometido a este procedimiento durante el periodo inmediato y mediato, basados en evidencia científica.

La EA sin cirugía se asocia con una pobre supervivencia una vez que los síntomas se desarrollan (1). Constituye la enfermedad estructural más frecuente en personas de edad avanzada con una incidencia de entre el $15 \%$ y el $20 \%$, que en octogenarios alcanza valores cercanos al $50 \%$ (2), particularmente la de etiología degenerativa. La sustitución valvular aórtica quirúrgica es el tratamiento de elección en pacientes con EA severa. Sin embargo, la morbilidad y mortalidad que tiene este grupo de población resulta en un riesgo bastante elevado. De acuerdo con los datos de Euro Heart Survey, hasta un tercio se rechaza para cirugía por presentar excesivas comorbilidades asociadas o expectativa de vida corta (3). Ante este contexto, se han diseñado prótesis valvulares aórticas que se implantan a través de catéteres y constituyen actualmente una alternativa terapéutica en estos casos (4).

\section{Estenosis valvular aórtica}

La EA es la obstrucción de la eyección ventricular izquierda localizada en la válvula aórtica; se desarrolla de forma gradual, lo que permite que el ventrículo izquierdo se adapte a la sobrecarga de presión sistólica e incremente su grosor parietal, y de esta manera venza el aumento de la poscarga para mantener el volumen minuto cardiaco. El área valvular aórtica normalmente es de $3 \mathrm{~cm}^{2}$ a $4 \mathrm{~cm}^{2}$ y comienza a producir un gradiente de presión cuando se reduce; por lo tanto, se denomina estenosis ligera cuando la reducción es de $1,5 \mathrm{~cm}^{2}$; moderada, entre $1,5 \mathrm{~cm}^{2}$ y $1 \mathrm{~cm}^{2}$, y si el área es menor de $1 \mathrm{~cm}^{2}$, se considera severa (5), lo cual causa un obstáculo a la expulsión sanguínea del ventrículo izquierdo.

Los factores que intervienen en la gravedad de la estenosis son principalmente cuatro: el área valvular aórtica con un gradiente promedio de $\geq 40 \mathrm{~mm} \mathrm{Hg}$ o un área valvular aórtica de $\leq 1 \mathrm{~cm}^{2}$, el gasto sistólico, la contractilidad del miocardio y el tiempo de evolución de la estenosis aórtica (6). En los pacientes con bajos gradientes transaórticos, aun cuando tengan un área valvular aórtica consistente con EA severa, la ecocardiografía de estrés con dosis bajas de dobutamina ayudan a distinguir un estadio verdaderamente grave de la seudograve (7).

La EA es de diversa etiología; prevalece del $6 \%$ al $40 \%$ la de origen congénito, y así es la más frecuente es la aorta bicúspide y casi siempre es un $2-11 \%$, es resultado de la cicatrización de la válvula aórtica y la adhe- 
rencia de las comisuras calcificadas de las valvas, secundaria a infección por estreptococo B-hemolítico del grupo A y se asocia con enfermedad valvular mitral (8). Por último, la EA degenerativa o senil se presenta en el $50 \%$ al $70 \%$ de los casos cuando las valvas están inmovilizadas por depósitos de calcio a lo largo de las líneas de flexión en sus bases. En pacientes de edad avanzada es frecuente encontrar EA muy grave asintomática, probablemente secundaria a una vida sedentaria con actividad física bastante limitada. Asimismo, no es raro que las personas padezcan otras enfermedades concomitantes como obesidad, insuficiencia respiratoria, osteoartritis, entre otras (9).

Los sintomas característicos de la EA severa son angina, síncope e insuficiencia cardiaca. Aproximadamente, el 35\% de los enfermos debuta con angina como primer sintoma, el 15\% lo hace con síncope de esfuerzo y en el $50 \%$ de los pacientes la insuficiencia cardiaca es la primera manifestación (10). La angina de pecho es consecuencia de un desequilibrio entre la demanda miocárdica de oxígeno y el aporte coronario de este. Se presenta en todos los grupos de edad y solo aproximadamente el $50 \%$ de los pacientes tiene arterias coronarias con obstrucción significativa. E1 síncope se debe a la reducción de flujo sanguíneo cerebral que se produce durante el esfuerzo cuando la presión arterial disminuye en presencia de vasodilatación periférica con gasto cardiaco fijo por la obstrucción. La disnea es el sintoma que con mayor frecuencia se presenta en la insuficiencia cardiaca; es la expresión del aumento de la presión venocapilar pulmonar, y se manifiesta primero como disminución de la tolerancia al esfuerzo y posteriormente como disnea de decúbito, paroxística y nocturna. En ocasiones, la primera manifestación es el edema agudo de pulmón (6).

\section{Implante valvular percutáneo transcatéter}

La cirugía de remplazo valvular aórtico es el tratamiento de elección en estos pacientes, ya que deriva en un alivio efectivo de los síntomas y en un aumento de la supervivencia; no obstante, con el desarrollo de la tecnologia, recientemente se ha introducido la técnica de implantación de prótesis biológicas por vía percutánea para aquellos pacientes que no son candidatos a la cirugía, conocido como TAVI, el cual ofrece la ventaja de implantar una prótesis valvular en el interior de la válvula aórtica del corazón nativo, sin tener que recurrir a la incisión quirúrgica (11).

Al inicio, el sistema del catéter percutáneo para el tratamiento de la EA fue probado en modelos animales en la década de los sesenta (12). Durante los siguientes años se desarrolló la tecnología del stent (endoprótesis vascular), que fue necesaria antes de la primera implantación de la prótesis transcatéter. En el 2000, Bonhoeffer y su grupo de colaboradores realizaron el primer implante exitoso de válvula cardiaca en humanos en un niño de 12 años de edad con estenosis e insuficiencia del conducto protésico valvulado, desde el ventrículo derecho a la válvula pulmonar (13). Dos años más tarde, Criber y colaboradores efectuaron el primer implante exitoso con una bioprótesis de pericardio bovino montada dentro de un 
stent con un balón expandible en un paciente con estenosis severa, a través del acceso anterógrado transeptal (14).

En el 2005, Grube y su equipo publicaron el primer reporte de implante percutáneo exitoso por vía retrógrada femoral de una novedosa bioprótesis valvular aórtica autoexpandible y parcialmente reposicionable (15). Entre los diferentes registros publicados, existe una consistencia de éxito entre el $95 \%$ y $99 \%$, con una notable mejoría hemodinámica inmediata y a los 30 días, tanto en el aumento del área valvar como en el descenso del gradiente. También se observó mejoría clínica, por lo menos en una clase funcional del $80 \%$ de los pacientes. Evidentemente, lo anterior se acompañó con mejoría en su calidad de vida, incluyendo síntomas, limitaciones físicas y sociales (16).

Actualmente existen dos prótesis valvulares en el mercado: la válvula Core Valve ${ }^{\circledR}$ (Medtronic Inc., Minneapolis, Minnesota), que es un dispositivo trivalvular biológico de pericardio porcino, montado y suturado en un marco de stent autoexpansible de nitinol (figura 1). Existen dos tamaños de dispositivos para las distintas dimensiones de anillo aórtico: una de $26 \mathrm{~mm}$ y otra de $27 \mathrm{~mm}$, y de recién ingreso, una de $31 \mathrm{~mm}$ (17), para su inserción retrógrada a través de una arteria femoral/subclavia/axilar o un acceso transaórtico directo (14).

El otro dispositivo es el Edwards Sapiens ${ }^{\circledR}$ (Edwards Lifesciences, Irvine, California), que utiliza una válvula de pericardio bovino suturado a

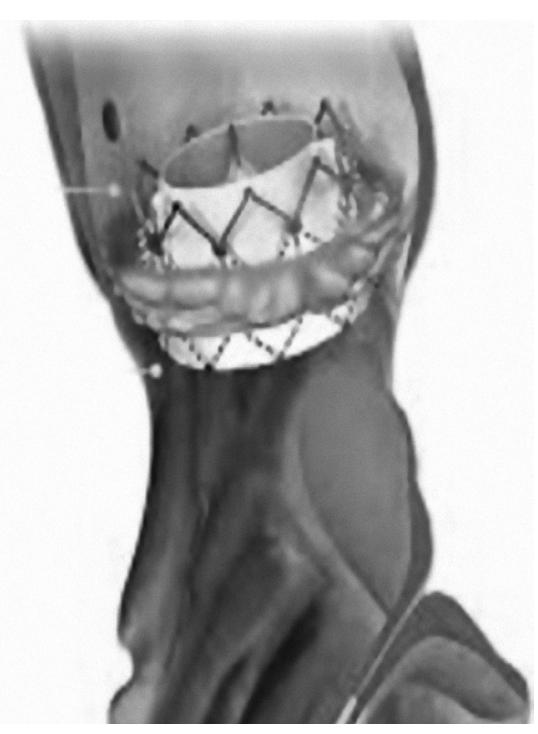

Figura 1. Válvula cardiaca transcatéter Edwards Sapien XT® 
un stent de marco metálico. La implantación de la prótesis se lleva a cabo por el inflado del balón. Existen tres tamaños de anillos 23, 26 y 29 mm (figura 2) (18). Se puede utilizar en accesos de forma anterógrada (transapical) o retrógrada (tranfemoral, transsubclavia o transaórtica). Aunque también se han implantado por la arteria pulmonar o dentro de una prótesis en la aorta degenerada (19), incluso existen otros dispositivos en etapas finales de estudio clínico (20).

\section{Indicaciones y contraindicaciones del TAVI}

El TAVI está indicado para pacientes con síntomas severos de EA. La confirmación de la gravedad es obligatoria en todos los casos (21); así mismo, está contemplado en quienes está prohibida la cirugía convencional o los que tienen alto riesgo quirúrgico, que puede ser valorado por la escala EuroSCORE (22) o STS (23). Aun cuando muchos pacientes satisfacen los criterios de inclusión para el TAVI, el procedimiento no es viable para todos. Con la evolución de la tecnología y la experiencia de los médicos, se han ido descartando algunas contraindicaciones mientras que otras han emergido. Entre los pacientes que no son candidatos para el tratamiento con TAVI se encuentran aquellas personas $\geq$ de 65 años de edad, que cuenten con la presencia de algunas comorbilidades como: cirrosis hepática, insuficiencia renal, insuficiencia pulmonar, previa cirugía cardiaca, hipertensión pulmonar, embolismo pulmonar recurrente o falla de ventrículo derecho (24).

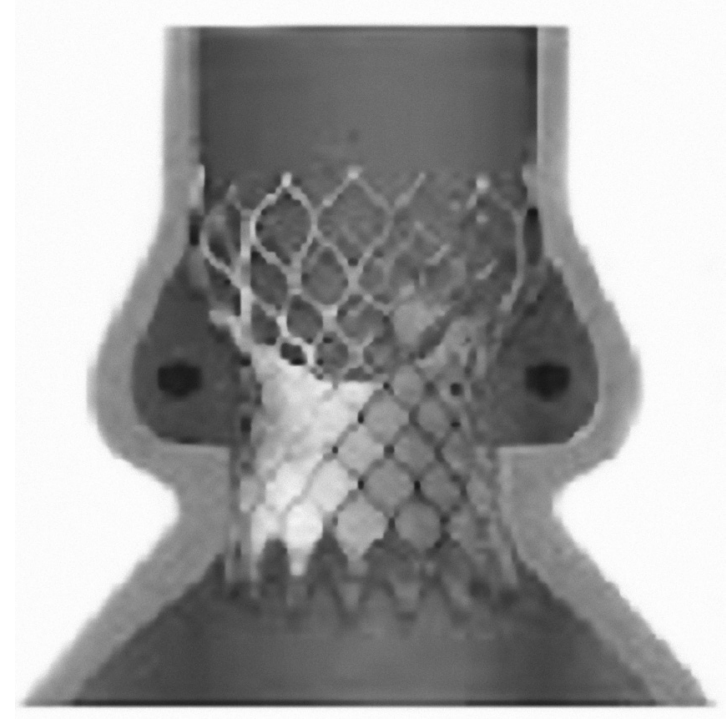

FigURA 2. Válvula cardiaca transcatéter CoreValve ${ }^{\circledR}$ System

Fuente://www.INTRAMED.NET/CONTENIDOVER.ASP?CONTENIDOID=72375\&PAGINA=36 
Algunas otras contraindicaciones son la presencia de calcificación valvular altamente asimétrica, lo cual puede comprimir algún vaso durante el procedimiento; la aorta bicúspide, debido al riesgo de expansión incompleta de la prótesis; anillo aórtico menor de $18 \mathrm{~mm}$ o superior de $25 \mathrm{~mm}$; fracción de eyección del ventrículo izquierdo menor del $20 \%$; enfermedades de la arteria coronaria que requieran revascularización; insuficiencia respiratoria severa, previo accidente cerebrovascular, e insuficiencia renal severa (24).

\section{Descripción del procedimiento del TAVI}

La vía transfemoral es el acceso de primera elección más común para el despliegue del dispositivo de la válvula aórtica transcatéter, debido a su mínima naturaleza invasiva; sin embargo, existe una tasa mayor de hemorragias y embolizaciones cerebrales en pacientes con estenosis aortoiliaca, tortuosidad, ateroesclerosis y aquellos con pequeñas venas. En estos casos, el acceso alternativo es el aórtico, el transapical, el subclavio o por la arteria axilar (25).

El procedimiento se realiza bajo anestesia general y sedación, en un ambiente estéril en el servicio de hemodinamia, en la mayoría de los casos con el paciente intubado. Se coloca un cable de marcapasos temporal a través de la vena femoral en el ventrículo derecho y la valvuloplastia aórtica con balón se realiza bajo la estimulación ventricular rápida con una frecuencia de entre 180 y 200 latidos por minuto (26).

Una vez realizada la valvuloplastía, la vaina se introduce por la dilatación secuencial del sitio de acceso de la arteria femoral a la aorta, y a través de este se inserta la prótesis del balón montado. El sistema se hace avanzar a través de la válvula nativa y el globo es inflado. De nuevo, bajo la estimulación ventricular rápida, se comprime la válvula para expandir el stent e implantarlo en el anillo de la válvula nativa. El procedimiento se controla por angiografía y la ecografía transesofágica.

Los dispositivos de cierre para obtener una hemostasia arterial posterior a una intervención percutánea pueden ser: a) Prostar-XL ${ }^{\circledR}$ (Abbott, Estados Unidos), dispositivo diseñado para el cierre de punciones de hasta 10 F mediante suturas en el sitio de inserción arterial al término de procedimientos endovasculares (27). Se basa en un mecanismo relativamente complejo que implica la liberación de 4 agujas desde el interior hacia el exterior de la arteria (28). b) Angio-Seal ${ }^{\circledR}$ (St. Jude Medical, St. Paul, Minnesota) es otro dispositivo constituido por un componente intravascular que consiste en una estructura utilizada como anclaje, y otro extravascular de tamaño pequeño hecho de colágeno bovino, situado por fuera de la arteriotomía y una sutura que mantiene los componentes unidos. La hemostasia se logra en el lugar de punción cuando el componente de colágeno se presiona contra la adventicia de la pared vascular y el componente intravascular mantiene al dispositivo fijo desde la parte luminar de la pared vascular $(29,30)$.

\section{Complicaciones del TAVI}

Los eventos cerebrovasculares se han convertido en una de las complicaciones más importantes del TAVI (31), y pese a que se han reportado tasas inferiores al $5 \%$ en la mayoría de los casos, estas cifras son bastante bajas 
teniendo en cuenta la edad avanzada y la alta prevalencia de ateroesclerosis de los pacientes sometidos al TAVI (20). La incidencia de los eventos fue asociada con la vía de acceso, donde se observó la tasa más baja en la arteria transapical (2,7\%) (21). Aunque los eventos cerebrovasculares se manifiestan claramente en los sintomas clínicos, se han demostrado con estudios de resonancia magnética cuadros de isquemia cerebral silentes (32) en entre el $66 \%$ y el $84 \%$ de los pacientes, posterior al implante de la prótesis, independientemente del tipo de válvula utilizada y el abordaje (33).

A pesar del avance de la tecnología de las válvulas en cuanto a la disminución gradual de la vaina y del tamaño del catéter, las principales determinantes de las complicaciones vasculares subyacen en el alto riesgo de la población tratada, el pequeño diámetro y la tortuosidad de los vasos. Es de resaltar que el acceso transfemoral está asociado con el mayor número de riesgos en comparación con el acceso transapical (21). The Valve Academic Research Consortium define las principales complicaciones vasculares en mayores y menores $(34,35)$ (tabla 1$)$.

TABLA 1. Complicaciones vasculares en el TAVI

\begin{tabular}{|c|c|}
\hline Mayores & Menores \\
\hline $\begin{array}{l}\text { Cualquier disección aórtica, ruptura de } \\
\text { la aorta, ruptura del anillo, perforación } \\
\text { del ventrículo izquierdo o un aneurisma/ } \\
\text { seudoaneurisma apical. }\end{array}$ & $\begin{array}{l}\text { Acceso al sitio o lesión vascular relacio- } \\
\text { nada con el acceso, que no conducen a } \\
\text { la muerte o son potencialmente mortales } \\
\text { o hemorragia grave, isquemia visceral o } \\
\text { deterioro neurológico. }\end{array}$ \\
\hline $\begin{array}{l}\text { Acceso al sitio o lesión vascular relaciona- } \\
\text { da con el acceso (disección, estenosis, per- } \\
\text { foración, ruptura, fistula arteriovenosa, } \\
\text { hematomas, lesiones irreversibles en los } \\
\text { nervios, síndrome compartimental, fallo } \\
\text { en el dispositivo de cierre percutáneo), que } \\
\text { conducen a la muerte o ponen en peligro } \\
\text { la vida o una hemorragia grave, isquemia } \\
\text { visceral o deterioro neurológico. }\end{array}$ & $\begin{array}{l}\text { La embolización distal tratado con em- } \\
\text { bolectomía o trombectomía y no cause } \\
\text { la amputación o daño irreversible de } \\
\text { órganos. }\end{array}$ \\
\hline $\begin{array}{l}\text { La embolización distal (no cerebral) de } \\
\text { una fuente vascular que requiera cirugía } \\
\text { o que cause la amputación del miembro o } \\
\text { daños irreversibles a los órganos. }\end{array}$ & $\begin{array}{l}\text { Cualquier colocación de stent endovascu- } \\
\text { lar no planificado o una intervención qui- } \\
\text { rúrgica no planificada que no cumplan } \\
\text { con los criterios de una mayor complica- } \\
\text { ción vascular. }\end{array}$ \\
\hline $\begin{array}{l}\text { El uso endovascular no planificado, una } \\
\text { intervención quirúrgica asociada con } \\
\text { la muerte, hemorragia grave, isquemia } \\
\text { visceral, deterioro neurológico. }\end{array}$ & $\begin{array}{l}\text { Reparación vascular o la necesidad de } \\
\text { reparación (a través de la cirugía de com- } \\
\text { prensión guiada por ecografía, la emboli- } \\
\text { zación transcáteter o stent-graft). }\end{array}$ \\
\hline \multicolumn{2}{|l|}{$\begin{array}{l}\text { Isquemia de las extremidades inferio- } \\
\text { res documentado por los sintomas del } \\
\text { paciente, el examen físico o la disminu- } \\
\text { ción o ausencia de flujo sanguíneo en las } \\
\text { extremidades inferiores. }\end{array}$} \\
\hline \multicolumn{2}{|l|}{$\begin{array}{l}\text { Cirugía de las lesiones o los nervios rela- } \\
\text { cionados con el sitio de acceso. }\end{array}$} \\
\hline $\begin{array}{l}\text { Lesión nerviosa relacionada con el sitio } \\
\text { de acceso permanente. }\end{array}$ & \\
\hline
\end{tabular}


Asimismo, la etiología de falla renal después del TAVI es reconocida por una combinación de múltiples factores como arteriopatía, hipertensión; así como la edad fisiológica, que está relacionada con la disminución de las nefronas, antecedentes de disfunción renal y diabetes, o bien está asociada con el medio de contraste utilizado en el procedimiento, o varios momentos de hipotensión (36).

El bloqueo auriculoventricular es de las complicaciones más frecuentes, lo que resulta en una alta incidencia de colocación de marcapasos definitivo postimplante valvular (37). De acuerdo con un estudio realizado, en el 39,3\% de los pacientes se debió a bloqueo auriculoventricular de tercer grado y se observó que la tasa más alta se presentó en el grupo del dispositivo Core Valve ${ }^{\circledR}$ respecto con el de Edwards Sapiens ${ }^{\circledR}$ (37). Las razones de la necesidad de un marcapasos aún no están claras, pero pueden estar relacionadas con el hecho de que esta última válvula es más corta que la primera (38).

Así mismo, Piazza y cols. fueron los primeros en sugerir que la ocurrencia de alteraciones de la conducción está asociada con la posición de la implantación de la prótesis durante el procedimiento con el dispositivo CoreValve ${ }^{\circledR}$ (39). Se especula que la lesión del tejido de conducción durante el TAVI es inducida por presión mecánica por el sistema de conducción de la prótesis (39); en un análisis más reciente, los mismos autores encontraron que los siguientes son factores predictivos para el bloqueo de rama izquierda: género masculino, previo infarto del miocardio y bloqueo de rama preexistente (40).

\section{Metodología}

Con base en la pregunta ¿cuáles son los principales cuidados de enfermería ante el implante de la válvula aórtica por vía percutánea?, se realizó una búsqueda sistematizada de la literatura en idioma inglés y español dentro del periodo octubre-diciembre del 2013 con los términos MeSh TAVI, estenosis aórtica, implante percutáneo, cuidados de enfermería, valvuloplastia, cateterismo cardiaco, complicaciones transcatéter, aortic stenosis, valve implantation, aortic transcatheter, valve percutaneous, transcatether complications. Se utilizaron las bases de datos PubMed, EMBASE, SciELO, Medigraphic, Cochrane y Biblioteca Virtual en Salud, en las cuales se encontraron y revisaron estudios de caso clínico, investigaciones, revisiones sistematizadas y metanálisis. La bibliografía encontrada en español fue proveniente principalmente de España y América Latina; no obstante, solo se encontraron tres manuscritos del área de enfermería, dos en idioma inglés y uno en español.

Los criterios de selección fueron artículos completos que abordaron el tema de implante percutáneo transcatéter, donde la población de estudio estuviera en la octava década de la vida o más, sin importar el género. Se hizo hincapié en la búsqueda de artículos que incluyeran posibles complicaciones postimplante. Las publicaciones seleccionadas fueron de enero del 2007 a diciembre del 2013. 


\section{Cuidado del paciente posterior al TAVI}

El profesional de enfermería desempeña un papel fundamental en la recuperación de la persona a quien se le ha implantado la válvula aórtica transcatéter. Los conocimientos actuales y sustentados en evidencia científica sobre las posibles complicaciones y los cuidados posteriores aportan seguridad y calidad al proceso asistencial (40). En la tabla 2 se presentan las principales intervenciones de enfermería.

TABLA 2. Intervenciones de enfermería pos-TAVI

\begin{tabular}{|c|c|c|}
\hline Tiempo & Valoración & Intervención \\
\hline \multirow{4}{*}{$\begin{array}{l}\text { 1-2 horas } \\
\text { posteriores al } \\
\text { procedimiento }\end{array}$} & $\begin{array}{l}\text { Evaluación } \\
\text { general } \\
\text { después de la } \\
\text { anestesia }\end{array}$ & Toma, registro y valoración de constantes vitales $(4,41)$. \\
\hline & $\begin{array}{l}\text { Monitoreo } \\
\text { cardiaco }\end{array}$ & $\begin{array}{l}\text { Realizar electrocardiograma (ECG) de } 12 \text { de- } \\
\text { rivaciones tras la implantación del marcapa- } \\
\text { so, previa verificación de instalación adecua- } \\
\text { da mediante placa de rayos X de tórax (42). } \\
\text { Evaluar el ECG. Observar si las espigas de marca- } \\
\text { paso ocurren demasiado cerca de la onda T (puede } \\
\text { producir taquicardia o fibrilación ventricular) (43). } \\
\text { Verificar que el ritmo y frecuencia cardia- } \\
\text { ca sean compatibles con los parámetros esta- } \\
\text { blecidos con el generador del marcapaso. } \\
\text { Indicar al paciente que comunique cualquier sín- } \\
\text { toma como mareo, palpitaciones o dolor (43); } \\
\text { esto puede estar relacionado con falla o disfun- } \\
\text { ción del marcapaso. O bien, hipo, debido a la } \\
\text { estimulación muscular diafragmática (44). }\end{array}$ \\
\hline & $\begin{array}{l}\text { Dolor del } \\
\text { miembro pun- } \\
\text { cionado }\end{array}$ & $\begin{array}{l}\text { Valorar la intensidad del dolor mediante la es- } \\
\text { cala análoga-visual (45). Incluir características, } \\
\text { duración, frecuencia, calidad y factores desenca- } \\
\text { denantes. En caso de prescripción médica de anal- } \\
\text { gésico, tomar en cuenta los siete correctos (46). }\end{array}$ \\
\hline & $\begin{array}{l}\text { Estado del } \\
\text { apósito }\end{array}$ & $\begin{array}{l}\text { Revisar cada } 30 \text { minutos durante las dos pri- } \\
\text { meras horas y posteriormente cada seis has- } \\
\text { ta la retirada de este; se observará y registrará } \\
\text { tipo, cantidad y color de la sangre del apósito. } \\
\text { Este se puede retirar a las } 24 \text { horas (47). }\end{array}$ \\
\hline
\end{tabular}




\begin{tabular}{|c|c|c|}
\hline Tiempo & Valoración & Intervención \\
\hline \multirow{4}{*}{$\begin{array}{l}24 \text { horas } \\
\text { posteriores al } \\
\text { procedimiento }\end{array}$} & $\begin{array}{l}\text { Sitio de inser- } \\
\text { ción vascular }\end{array}$ & $\begin{array}{l}\text { Observar en busca de presencia enrojeci- } \\
\text { miento, aumento de temperatura, equimosis, } \\
\text { sangrado, salida de material purulento. }\end{array}$ \\
\hline & $\begin{array}{l}\text { Movilidad } \\
\text { del miembro } \\
\text { puncionado }\end{array}$ & $\begin{array}{l}\text { Durante este periodo la extremidad afecta- } \\
\text { da debe ser elevada a no más de } 30^{\circ}(48) \text {. } \\
\text { Es importante que el paciente no flexio- } \\
\text { ne ni movilice la extremidad (49). }\end{array}$ \\
\hline & \begin{tabular}{|l|} 
Concen- \\
tración de \\
hemoglobina \\
y plaquetas \\
\end{tabular} & $\begin{array}{l}\text { Toma de muestras de laboratorio después del } \\
\text { procedimiento y los dias siguientes. La dismi- } \\
\text { nución de las plaquetas, de la hemoglobina y del } \\
\text { hematocrito pueden indicar sangrado (42). }\end{array}$ \\
\hline & $\begin{array}{l}\text { Datos de } \\
\text { complicacio- } \\
\text { nes del acceso } \\
\text { vascular }\end{array}$ & $\begin{array}{l}\text { Detectar oportunamente si presenta isquemia del } \\
\text { miembro puncionado mediante presencia e intensidad } \\
\text { de pulsos, edema, disminución de temperatura, palidez } \\
\text { con cianosis o sin esta, o bien eritrosis y trofismo } \\
\text { del pie }(42,50) \text {. Preguntar al paciente si tiene sensa- } \\
\text { ción de hormigueo o entumecimiento del miembro. } \\
\\
\text { Debido a la punción de la arteria femoral, el pacien- } \\
\text { te puede presentar acumulación de sangre en el } \\
\text { retroperitoneo. Generalmente se presenta con dis- } \\
\text { tensión abdominal, dolor lumbar y disminución del } \\
\text { hematocrito (51). Estar atentos a estos síntomas. }\end{array}$ \\
\hline \multirow{3}{*}{$\begin{array}{l}48 \text { horas } \\
\text { posteriores al } \\
\text { procedimiento }\end{array}$} & $\begin{array}{l}\text { Alteraciones } \\
\text { neurológicas }\end{array}$ & $\begin{array}{l}\text { Observar el tamaño pupilar y reflejo de movimientos } \\
\text { motores o respuestas verbales mediante las escalas } \\
\text { de coma de Glasgow y la escala de Cincinnati }(42,43) \\
\text { y la fuerza muscular mediante la escala de valora- } \\
\text { ción muscular del Medical Research Council }(52) \text {. }\end{array}$ \\
\hline & $\begin{array}{l}\text { Evaluar fun- } \\
\text { ción renal }\end{array}$ & $\begin{array}{l}\text { Se debe cuantificar el volumen urinario (>1 } \\
\mathrm{ml} / \mathrm{kg} / \mathrm{h}) \text {, concentraciones de creatinina }(0,7- \\
1,5 \mathrm{mg} / \mathrm{dl}), \text { nitrógeno ureico }(9-20 \mathrm{mg} / \mathrm{dl}), \\
\text { cantidades plasmáticas de electrolitos (K 3,9- } \\
5,6 \mathrm{mEq} / \mathrm{L}, \mathrm{Na} 135-155 \mathrm{mEq} / \mathrm{L}, \mathrm{Cl} 95-106 \\
\mathrm{mEq} / \mathrm{L}),(53,54), \text { ya que la alteración de al- } \\
\text { guno de ellos puede indicar falla renal. } \\
\\
\text { Valorar continuamente en el monitor ondas T tocan- } \\
\text { do techo, ondas P anchas y planas o la pérdida de } \\
\text { las ondas P y el ensanchamiento del complejo QRS } \\
\text { (46), sugerentes de niveles elevados de potasio. }\end{array}$ \\
\hline & $\begin{array}{l}\text { Dolor del } \\
\text { miembro pun- } \\
\text { cionado }\end{array}$ & $\begin{array}{l}\text { Valorar nuevamente el dolor como } \\
\text { se realizó anteriormente. }\end{array}$ \\
\hline
\end{tabular}




\begin{tabular}{|c|c|c|}
\hline Tiempo & Valoración & Intervención \\
\hline \multirow{5}{*}{ Previo al alta } & $\begin{array}{l}\text { Evaluación } \\
\text { cognitiva }\end{array}$ & $\begin{array}{l}\text { Aplicar la escala Mini-Mental State Examination } \\
\text { (MMSE) de Folstein o cualquier otra de su tipo, para } \\
\text { una valoración inicial de alteraciones cognitivas } \\
\text { (55). De esta manera se podrá adecuar la informa- } \\
\text { ción sobre su autocuidado en casa y, de ser nece- } \\
\text { sario, hacer corresponsable al cuidador primario. }\end{array}$ \\
\hline & $\begin{array}{l}\text { Antiagregan- } \\
\text { tes plaqueta- } \\
\text { rios }\end{array}$ & $\begin{array}{l}\text { Habitualmente, el antiagregante plaquetario } \\
\text { es prescrito por el médico durante los } 6 \text { me- } \\
\text { ses después del procedimiento (56). Explicar } \\
\text { al paciente que debe establecer un horario fijo } \\
\text { y de preferencia entre los alimentos (57). } \\
\text { Es importante explicar al paciente que disminuya } \\
\text { alimentos ricos en vitamina K (aguacate, brócoli, } \\
\text { espinacas, coliflor, lechuga, yema de huevo, soya y } \\
\text { todos sus derivados, hígado de res y cerdo, té verde), ya } \\
\text { que pueden alterar los efectos de la anticoagulación. }\end{array}$ \\
\hline & $\begin{array}{l}\text { Verificación } \\
\text { del dispositivo }\end{array}$ & $\begin{array}{l}\text { Es de considerar el paciente se vaya de alta con la } \\
\text { solicitud para realizarle un ecocardiograma den- } \\
\text { tro del primer mes, para verificar la colocación } \\
\text { del dispositivo dentro de la válvula aórtica nativa, } \\
\text { así como su buen funcionamiento, especialmente } \\
\text { para evaluar si presenta fuga paravalvular (58). }\end{array}$ \\
\hline & $\begin{array}{l}\text { Rehabilita- } \\
\text { ción cardiaca }\end{array}$ & $\begin{array}{l}\text { Durante la hospitalización (fase I) se realizarán in- } \\
\text { tervenciones encaminadas a contrarrestar los efectos } \\
\text { del reposo prolongado: mantener el tono muscu- } \\
\text { lar, prevenir la hipotensión postural y disminuir el } \\
\text { riesgo de trombosis venosa profunda. Así mismo, es } \\
\text { pertinente informar al paciente y su familia sobre } \\
\text { la dieta adecuada, medicamentos prescritos, activi- } \\
\text { dades físicas permitidas y signos de alarma (59). }\end{array}$ \\
\hline & $\begin{array}{l}\text { Signos de } \\
\text { alarma }\end{array}$ & $\begin{array}{l}\text { Informar al paciente respecto a que debe estar atento } \\
\text { a signos de alarma que evidencien sangrado, como } \\
\text { derrames oculares, epistaxis, gingivorragia, hema- } \\
\text { temesis, hematuria, evacuaciones melénicas o con } \\
\text { sangre fresca, petequias, equimosis o hematomas } \\
\text { sin causas aparente (60). Igualmente, endocarditis } \\
\text { protésica manifestada por fiebre, astenia, mialgias } \\
\text { y artralgias u otros sintomas como pérdida de peso, } \\
\text { escalofríos, náusea, diaforesis nocturna o lumbalgia } \\
\text { (61). En caso de presentarse alguno de estos, acudir } \\
\text { inmediatamente al servicio de urgencias hospitalarias. }\end{array}$ \\
\hline
\end{tabular}

Fuente: elaboración propia 


\section{Conclusión}

Para poder ofrecer atención de calidad a las personas con TAVI es indispensable que el profesional de enfermería otorgue intervenciones específicas y especializadas de acuerdo con la evolución que se observe en el paciente. De esta forma, los cuidados se pueden ubicar en tres momentos:

a. Los cuidados inmediatos deberán basarse en una estricta valoración continua de las constantes vitales, el ritmo cardiaco, los parámetros sanguíneos que orienten a detectar desviaciones propias del procedimiento, así como estar pendientes de las relacionadas con la punción, las características del apósito oclusor, la movilidad del miembro pélvico afectado, así como detectar oportunamente complicaciones derivadas del acceso vascular.

b. Una vez que el paciente está más alerta y recordando que el evento cerebrovascular es una de las principales complicaciones, es imprescindible una valoración en busca de alteraciones neurológicas, mediante el uso de las diferentes herramientas para intervenir oportunamente. Además, brindar confort mediante la inhibición de malestares que afecten el equilibrio, especialmente el dolor; así como restablecer la movilidad para lograr independencia de la persona lo más rápido posible.

c. El papel del profesional en la integración del paciente a la vida cotidiana es importante, es decir, la planificación del alta. Debemos instruirle sobre los cuidados que debe aplicar en su domicilio, aclarando las dudas.

\section{Conflicto de interés}

Se declara no hubo ningún conflicto de interés.

\section{Referencias}

1. Reynolds MR, Magnuson EA, Lei Y, Leon MB, Smith CR, Svensson LG, et al. Healt-related quality of life after transcatheter aortic valve replacement in inoperable patients with severe aortic stenosis. Circulation [internet]. 2011[citado 7 oct 2013]; 124:[aprox. 7p]. Disponible en: circ.ahajournals.org/content/124/18/1964.long.

2. Piccinini F, Vrancic JM, Vaccarino G, Raich H, Siles G, Benzadón $\mathrm{M}$, et al. Cirugía de reemplazo valvular aórtico aislado en pacientes octogenarios: evaluación, riesgo operatorio y resultados a mediano plazo. Rev Argent Cardiol [internet]. 2010 [citado 9 oct 2013]; 78:[aprox. 8p]. Disponible en: http://www.scielo.org.ar/scielo.php?pid=S1850-37482010000600005\&script $=$ sci_arttetx.

3. Avanzas P, Muñoz GAJ, Segura J, Pan M, Alonso BJH, Lozano I, et al. Implante percutáneo de la prótesis valvular aórtica autoexpandible CoreValve ${ }^{\circledR}$ en pacientes con estenosis aórtica severa: experiencia inicial en España. Rev Esp. Cardiol [internet]. 2010 [citado 07 oct 2013]; 63 (2):[aprox. 6p]. Disponible en: www.revespcardiol. org/es/implante-percutaneo-valvula-autoexpandible-corevalve/articulo/90227054. 
4. Grando TA, Sarmento-Leite R, Lunardi P, Gomes CR, Specht F, Sarturi GA, et al. Anesthetic management and complications of percutaneous aortic valve implantation. Rev Bras Anestesiol [internet]. 2013 [citado 12 oct 2013];63(3):[aprox 7p]. Disponible en: http:// www.sciencedirect.com/science/article/pii/S0034709413702319.

5. Vélez H, Rojas W, Borrero J, Restrepo J. Cardiología. 6ª ed. Colombia: Corporación para las Investigaciones Biológicas; 2002.

6. Ortega MC, Puntunet M, Suarez MG, Leija C, Montesinos G, Cruz $\mathrm{G}$, et al. Guía de práctica clínica cardiovascular. México: Editorial Médica Panamericana; 2011.

7. Grupo de trabajo conjunto de la Sociedad Europea de Cardiología (ESC) y de la Asociación Europea de Cirugía Cardiotorácica (EACTS). Guías de práctica clínica sobre el tratamiento de las valvulopatías (versión 2012). Rev Esp Cardiol. 2013;66(2):131.e1-e42.

8. Teniza ND, Domínguez BA, Puntunet BML. Cuidado enfermero dirigido a la persona con estenosis aórtica. Rev Mex Enfer Cardiol. 2010;18(3):71-81.

9. Guadalajara JF. Cardiología. 7a ed. México: Méndez; 2013.

10. Sociedad Castellana de Cardiología. Lesiones valvulares. Monocardio. 2000;II(4):224-86.

11. Munkholm-Larsen S, Croce B, Harris R. TAVI: transcatheter aortic valve implantation. Ann Cardiothorac Surg [internet]. 2012 [citado 20 oct 2013];1(2):[aprox. 1p]. Disponible en: http://www.ncbi.nlm. nih.gov/pmc/articles/PMC3741751/.

12. Reinöhl J, von zurMühlen C, Moser M, Sorg S, Bode Ch, Zehender M. TAVI 2012: state of the art. J Thromb Thrombolysis [internet]. 2013 [citado 18 oct 2013];35:[aprox. 20p]. Disponible en: http:// link.springer.com/article/10.1007\%2Fs11239-012-0825-4.

13. León MB, Nikolsky E. The Next Revolution: Percutaneous aortic valve replacement. Rambam Maimonides Med J [internet]. 2010[citado 22 oct 2013];1(2):[aprox. 13p.]. Disponible en: htttp://www. ncbi.nlm.nih.gov/pmc/articles/PMC3678783/.

14. Généreux P, Head SJ, Wood DA, Kodali SK, Williams MR, Paradis $\mathrm{JM}$ et al. Transcatheter aortic valve implantation 10 year anniversary: review of current evidence and clinical implications. Eur Heart J [internet]. 2012 [citado 1 nov 2013];33:[aprox. 10 p.]. Disponible en: http://eurheartj.oxfordjournals.org/content/33/19/2388. long.

15. Hara H, Schwartz RS. Transcatheter aortic valve implantation in high-risk patients with severe aortic stenosis. Circ J [internet]. 2010 [citado 30 oct 2013];74:[aprox. 2 p.]. Disponible en: http:// www.jstage.jst.go.jp/article/circj/74/8/74_CJ-10-0557/_article.

16. XVII SOLACI Congress. Santiago de Chile; 3-6 de agosto de 2011. SOLACI. 2011. p. 2-21.

17. Caorsi C, Baeza Cr, Morís C, Oyonarte M, Venegas JC, Yovanovich $\mathrm{J}$, et al. Implante valvular aórtico transarterial: experiencia en Chi- 
le. Rev Med Chile [internet]. 2013 [citado 3 nov 2013];141: [aprox. 7 p.]. Disponible en: http://www.scielo.cl/scielo.php?script=sci_artt ext\&pid=S0034-98872013000100001.

18. Salinas P, Moreno R, Lopez-Sendon JL. Transcatheter aortic valve implantation: Current status an future perspectives. World J Cardiol [internet]. 2013 [citado 23 oct 2013]; 3(6):[aprox. 7 p.]. Disponible en: http://www.ncbi.nlm.nih.gov/pmc/articles/PMC3139039/.

19. Neragi MS, Michler RE. A review of most relevant complications of trancatheter aortic valve implantation. ISRN Cardiol [internet]. 2013 [citado 7 nov 2013]; [aprox. 15 p.]. Disponible en: http://www. ncbi.nlm.nih.gov/pmc/articles/PMC3703377/.

20. Molina MFJ, Lespron RMC. Manejo anestésico para el reemplazo de la válvula aórtica transcatéter. Rev Mex Anest [internet]. 2013 [citado 24 nov 2013];36 supl 1:[aprox. 3 p.]. Disponible en: http:// www.medigraphic.com/pdfs/rma/cma-2013/cmas131w.pdf.

21. Mylotte D, Martucci G, Piazza N. Patient selection for transcatheter aortic valve implantation: An interventional cardiology perspective. Ann Cardiothorac Surg [internet]. 2012 [citado 10 oct 2013];1(2):[aprox. 8 p.]. Disponible en: http://www.ncbi.nlm.nih. gov/pmc/articles/PMC3741729/.

22. Vavuranakis M, Voudris V, Vrachatis D, Thomopoulou S, Toutouzas K, Karavolias G, et al. Transcatheter aortic valve implantation, patient selection process and procedure: two centres' experience of the intervention without general anaesthesia. He1lenic J Cardiol [internet]. 2010 [citado 14 oct 2013];51:[aprox. 7 p.]. Disponible en: http://www.hellenicjcardiol.org/archive/full_ text/2010/6/2010_6_492.pdf.

23. Piazza N, Lange R, Martucci G, Serruys PW. Patient selection for trancatheter aortic valve implantation: patient risk profile and anatomical selection criteria. Arch Cardiovasc Dis [internet]. 2012 [citado 24 oct 2013];105:[aprox 7 p.]. Disponible en: http://www. sciencedirect.com/science/article/pii/S187521361200068X.

24. Leon MB, Smith CR, Mack M, Miller CD, Moses JF, Svensson LG, et al. Transcatheter aortic-valve implantation for aortic stenosis in patients who cannot undergo surgery. N Engl J Med [internet]. 2010 [citado 31 oct 2013];363:1597-1607. Disponible en: http:// www.nejm.org/doi/full/10.1056/NEJMoa1008232.

25. Sharma A, Arbab-Zadeh A, Dubey D, Shani J, Lazar J, Frankel R. Access site bleeding after transcatheter aortic valve implantation. J Thromb Thrombolysis [internet]. 2013 [citado 19 oct 2013];35:[aprox. 5 p.]. Disponible en: http://link.springer.com/article/10.1007\%2Fs11239-013-0928-6.

26. Bapat V, Attia R. Transaortic transcatheter aortic valve implantation: step-by-step guide. Semin Thoracic Surg [internet]. 2012 [citado 7 nov 2013];24:[aprox. 5 p.]. Disponible en: http://www. sciencedirect.com/science/article/pii/S1043067912000871. 
27. Zúñiga C, Mertens R, Valdés F, Krämer A, Mariné L, Bergoeing M, et al. Tratamiento percutáneo de aneurismas aorto-iliacos. Rev Chilena de Cirugía [internet]. 2008 [citado 15 nov 2013];60(5):[aprox. 4 p.]. Disponible en: http://www.scielo.cl/scielo.php?script=sci_ar ttext\&pid=S0718-40262008000500010.

28. Krajcer $Z$. Reparación del aneurisma aórtico abdominal percutáneo: estado actual e implicancias futuras. RACCV [internet]. 2011 [citado 26 nov 2013];IX(3):[aprox. 11 p.]. Disponible en: http://www. caccv.org.ar/raccv/V09-N3-2011-06.pdf.

29. Téllez A, Cheng Y, Wallace-Bradley D, Alviar C, Gallego C, Conditt $\mathrm{G}$, et al. Evaluación prospectiva in vivo del ritmo de degradación de dispositivo de cierre en angioplastia coronaria (AngioSeal). Rev Mex Cardiol [internet]. 2009 [citado 25 nov 2013];20(1):[aprox. 5 p.]. Disponible en: http://www.medigraphic.com/pdfs/cardio/h-2009/ h091e.pdf.

30. Mendéz J, Gil R, Millán FJ, Macias I, De Prada G, Ruiz C, et al. Implante percutáneo de prótesis valvular aórtica. Enferm Cardiol [internet]. 2010 [citado 28 nov 2013];XVII(49):[aprox. 3 p.]. Disponible en: http://www.enfermeriaencardiologia.com/revista/49_07.pdf.

31. Toggweiler S, Webb JG. Challenges in transcatheter aortic valve implantation. Swiss Med Wkly [internet]. 2012 [citado 29 nov 2013];142:[aprox. 4 p.]. Disponible en: http://www.smw.ch/content/ smw-2012-13735/.

32. Généreux P, Head SJ, Wood DA, Kodali SK, Williams MR, Paradis $\mathrm{J}-\mathrm{M}$, et al. Transcatheter aortic valve implantation: 10- year anniversary. Part II: clinical implications. Eur Heart J [internet]. 2012 [citado 23 nov 2013];33(pt 2):[aprox. 4 p.]. Disponible en: http:// eurheartj.oxfordjournals.org/content/33/19/2399.long

33. Paredes A, Martínez A. Prótesis valvular aórtica percutánea: ¿qué debemos saber? Rev Chil Cardiol [internet]. 2012 [citado 17 nov 2013]; 31:[aprox. 7 p.]. Disponible en: http://www.scielo.cl/scielo. php?pid=S0718-85602012000100008\&script=sci_arttext.

34. Finkelstein A, Birati EY, Abramowitz Y, Steinvil A, Sheinberg N, Biner S, et al. Transcatheter aortic valve implantation: A singlecenter experience of 300 cases. IMAJ [internet]. 2013 [citado 4 nov 2013]; 15:[aprox. 3 p.]. Disponible en: http://www.ima.org.il/IMAJ/ ViewArticle.aspx?year $=2013 \&$ month $=10 \&$ page $=613$.

35. Kappetein AP, Head SJ, Généreux P, Piazza N, Van Mieghem NM, Blackstone $\mathrm{EH}$, et al. Update standardized endpoint definitions for transcatheter aortic valve implantation: the valve Academic Research Consortium-2 consensus document. Eur Heart J [internet]. 2012 [citado 6 nov 2013];33:[aprox. 13 p.]. Disponible en: http://eurheartj.oxfordjournals.org/cgi/pmidlookup?view=long\&p $\operatorname{mid}=23026477$.

36. Franco A, Gerli C, Ruggeri L, Monaco F. Anesthesic management of transcatheter aortic valve implantation. Ann Card Anaesth [in- 
ternet]. 2012 [citado 10 nov 2013];15(1):[aprox. 7 p.]. Disponible en: http://www.annals.in/article.asp?issn=0971-9784;year=2012; volu me $=15$; issue $=1$; spage $=54 ;$ epage $=63$; aulast $=$ Franco .

37. Zahn R, Gerckens U, Grube E, Linke A, Sievert H, Eggebrecht H, et al. Transcatheter aortic valve implantation: first results from a multi-centre real-world registry. Eur Heart J [internet]. 2011 [citado 1 dic 2013];32:[aprox. 5 p.]. Disponible en: http://eurheartj. oxfordjournals.org/content/32/2/198.long.

38. Li X, Kong M, Jiang D, Dong A. Comparison 30-day clinical complications between transfemoral versus transapical aortic valve replacement for aortic stenosis: a meta-analysis review. J Cardiothorac Surg [internet]. 2013 [citado 27 nov 2013];8:186[aprox. 7 p.]. Disponible en: http://www.cardiothoracicsurgery.org/content/pdf/17498090-8-168.pdf.

39. D’Ancona G, Pasic M, Unbehaun A, Hetzer R. Permanent pacemarker implantation after transapical transcatheter aortic a valve implantation. Interact Cardiovasc Thorac Surg [internet]. 2011 [citado 25 nov 2013];13:[aprox. 3 p.]. Dsiponible en: http://icvts. oxfordjournals.org/content/13/4/373.full.pdf+html? sid=3567ccfO1abb-4eaf-bc9d-02b26a2b2d1c.

40. Galimany J, Díaz S, Pernas JC. Cuidados de enfermería al paciente sometido a cateterismo cardíaco y angioplastia coronaria. Enferm Cardiol [internet]. 2010 [citado 24 nov 2013];XVII(49):[aprox. 3 p.]. Disponible en: http://www.enfermeriaencardiologia.com/revista/49_11.pdf.

41. Lauck S, Mackay M, Galte C, Wilson M. A new option for the treatment of aortic stenosis: Percutaneous aortic valve remplacement. Crit Care Nurse [internet]. 2008 [citado 7 dic 2013];28(3):40-51. Disponible en: http://ccn.aacnjournals.org/content/28/3/40.long.

42. Álvarez GMJ, Arkáute EI, Belaustegi AA, Chaparro TS, Erice CA, González GMP, et al. Guía de práctica clínica: cuidados críticos de enfermería. s. 1.: Unidad de Medicina Intensiva del Hospital Txagorritxu; 2004.

43. Angell LM. Fast facts for the critical care nurse. New York: Springer Publishing Company; 2012.

44. Redecillas PMT, Cuadros GMJ, Gil RI, Herrero RI. Procedimientos de enfermería en la implantación de marcapaso trasvenosos temporal. NURE Inv [internet]. 2013 [citado 27 nov 2013];10(64):[aprox. 10 p.]. Disponible en: http://www.nureinvestigacion.es/protocolos detalle.cfm?id_PROTOCOLO=129\&id_menu=146.

45. Castillo BMD, Moreno PJP, Martínez PMV, Artiles SMM, Company SMC, García AMC, et al. Effectiveness of nursing intervention for adult patients experiencing chronic pain: a systematic review. JBI [internet]. 2010 [citado 7 dic 2013];8(28):[aprox. 20 p.]. Disponible 
en: http://www.evidenciaencuidados.es/evidenciaencuidados/pdf/ Intervenciones_en_dolor_cronico.pdf.

46. Ortega VMC, Suárez VMG, Jiménez VMC, Añorve GA, Cruz CM, Cruz AG, et al. Manual de evaluación de la calidad del servicio de enfermeria: estrategias para su aplicación. $3^{a}$ ed. México: Médica Panamericana; 2014.

47. Fonseca del Pozo FJ. Técnico en emergencias sanitarias. Madrid: Arán; 2009.

48. McRae ME, Rodger M, Bailey BA. Transcatheter and transapical aortic valve replacement. Crit Care Nurse [internet]. 2009 [citado 10 dic 2013];29(1):22-36. Disponible en: http://ccn.aacnjournals. org/content/29/1/22.long.

49. Serrano FJ, Martín A. Enfermedad arterial periférica: aspectos fisiopatológicos, clínicos y terapéuticos. Rev Esp Cardiol [internet]. 2007 [citado 13 dic 2013];60(9):[aprox. 15 p.]. Disponible en: http://www.revespcardiol.org/en/linkresolver/articulo-resolver/13114115/.

50. Nogales-Gaete J, Donoso A, Verdugo R, editores. Tratado de neurología clínica. Santiago de Chile: Editorial Universitaria; 2005.

51. Delgado SM, Gil HR, Márquez SA. Complicaciones vasculares postcateterismo según hemostasia. Enferm Cardiol [internet]. 2012 [citado 25 nov 2013];XIX(57): [aprox. 4 p.]. Disponible en: http://www. enfermeriaencardiologia.com/revista/57_04.pdf.

52. Puente ML, Valdazo M. Evaluación de la función muscular periférica. Arch Bronconeumol [internet]. 2001 [ citado 13 nov 2013];37:[aprox. 6 p.]. Disponible en: http://www.archbronconeumol.org/es/evaluacion-funcion-muscular-periferica/articulo/13014299/.

53. Schira M. Valoración renal y procedimientos diagnósticos. En Urden DL, Lough EM, Stacy MK. Cuidados intensivos en enfermería. Tomo II. Madrid: Océano; 2002.

54. Gennaro RA. Remington farmacia. 20a ed. Buenos Aires: Médica Panamericana; 2003.

55. Grupo de Trabajo de Neuropsicología Clínica de la Sociedad Neurológica Argentina. E1 "mini-mental state examination" en la Argentina: instrucciones para su administración. Rev Neurol Arg [internet]. 1999 [citado 21 dic 2013];24(1):[aprox. 5 p.]. Disponible en: http://www.imbiomed.com.mx/1/1/articulos. php? method=showDetail\&id_articulo=16084\&id_seccion=1318\&id_ ejemplar=1665\&id_revista $=95$.

56. Mancera MCS, López PA, Parra BA, Cortés VG. Anticoagulación vía oral. Rev Mex Enfer Cardiol. 2008;16(1):11-9.

57. Maldonado RNE. Atención al paciente con anticoagulantes. Rev Mex Enfer Cardiol. 2001;9(1-4):44-9.

58. Panos AM, George EL. Transcatheter aortic valve implantation options for treating severe aortic stenosis in the elderly: the nurse's 
role in postoperative monitoring and treatment. Dimens Crit Care Nurse [internet]. 2014 [citado 7 abril 2014];33(2): [aprox. 6 p.]. Disponible en: http://www.ncbi.nlm.nih.gov/pubmed/24496250.

59. Achury D, Rodríguez CSM, Agudelo CLA, Hoyos SJR, Acuña EJA. Calidad de vida del paciente con enfermedad cardiovascular que asiste al programa de rehabilitación cardiaca. Investig Enferm. Imagen Desarro [internet]. 2011 [citado 15 dic 2013];13(2):[aprox. 20 p.]. Disponible en: revistas.javeriana.edu.co/index.php/imagenydesarrollo/article/view/2977.

60. García J, Ruiz MJ, Zúñiga E. Protocolo de cuidados de enfermería al paciente sometido a cateterismo diagnóstico y terapéutico [internet]. Albacete: Complejo Hospitalario Universitario Albacete; 2013 [citado dic 2013]. Disponible en: http://www.chospab.es/publicaciones/proctocolosEnfermeria/documentos/f529d555a2540c7c48e6f7823693b272.pdf.

61. Reyes MM. Proceso enfermero a una persona con endocarditis infecciosa, aplicando los conceptos teóricos de Virginia Henderson. Rev Mex Enfer Cardiol. 2012;20(1):21-9. 